\title{
Synthesis and Properties of Nitrogen-Introduced Phenylazomethine Dendrimer
}

\author{
Ken Albrecht, ${ }^{1}$ Hideyuki Higashimura, ${ }^{2}$ and Kimihisa Yamamoto*1 \\ ${ }^{I}$ Chemical Resources Laboratory, Tokyo Institute of Technology, \\ 4259 Nagatsuta-cho, Midori-ku, Yokohama, Kanagawa 226-8503, Japan \\ ${ }^{2}$ Advanced Materials Research Laboratory, Sumitomo Chemical Co., Ltd., \\ 6, Kitahara, Tsukuba City, Ibaraki 300-3294, Japan \\ Email: yamamoto@res.titech.ac.jp
}

Supporting Information

Table of Contents

1. NMR spectra 

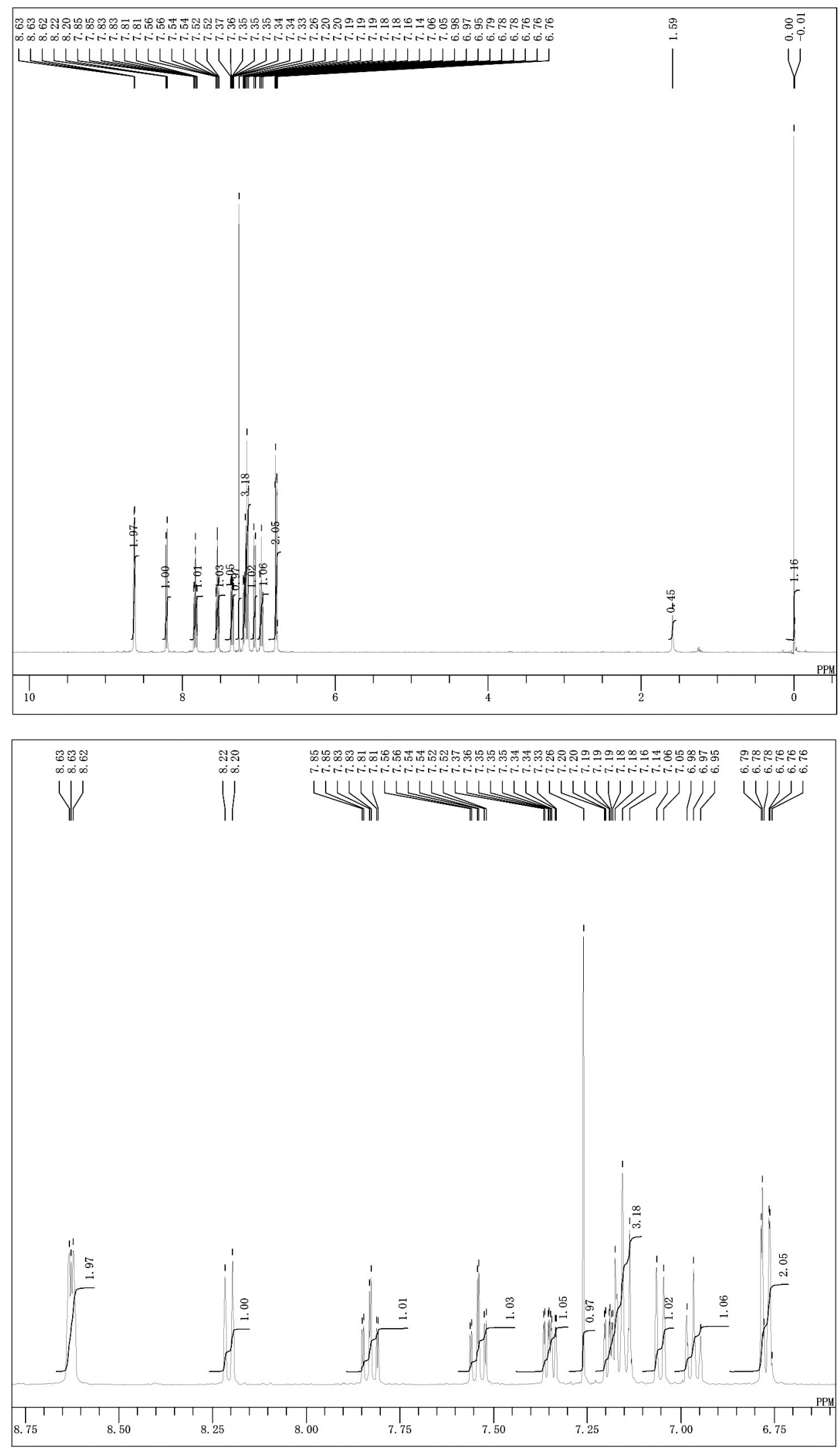

Figure S1. 1H NMR spectra of PyG0. 


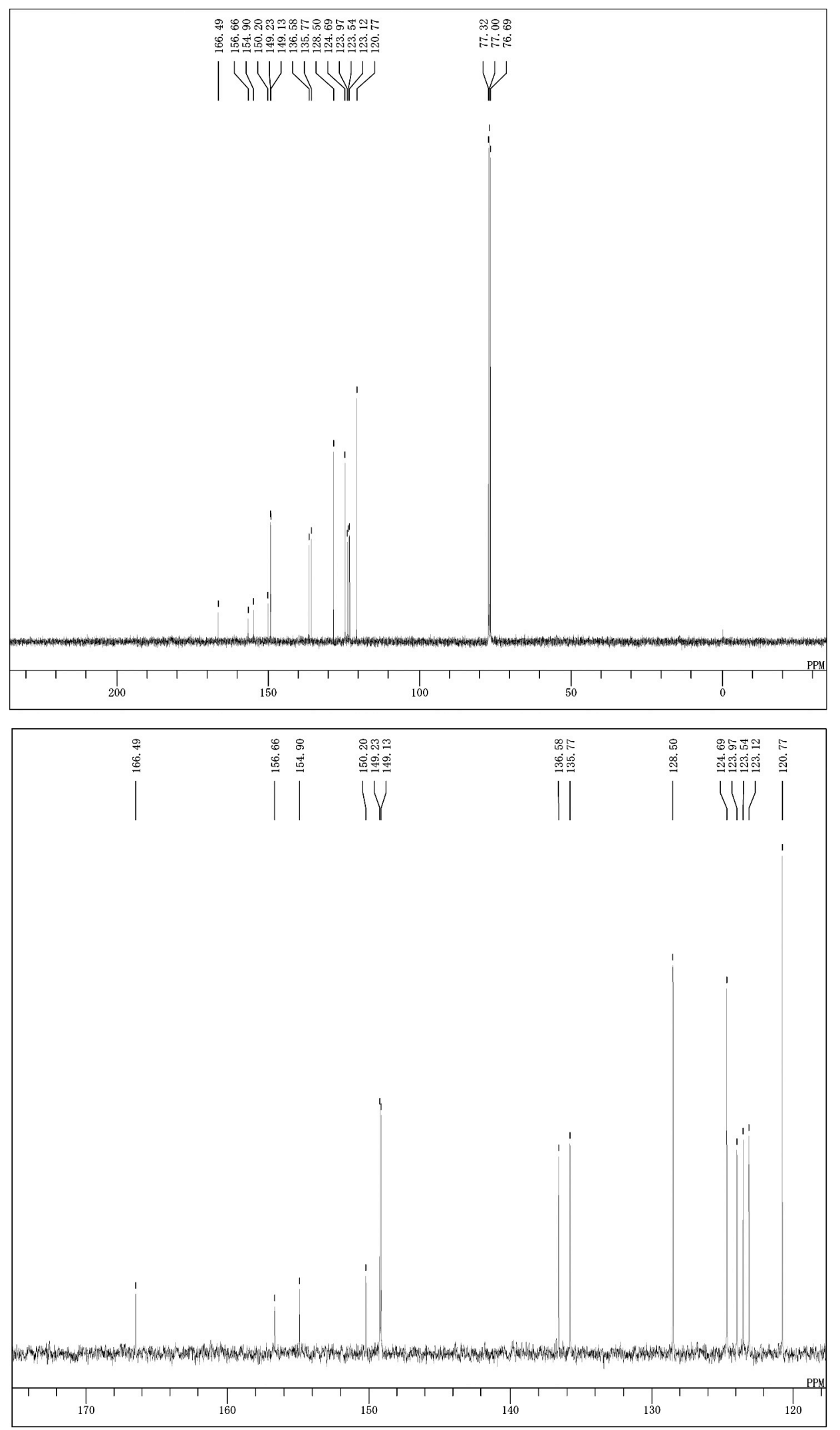

Figure S2. 13C NMR spectra of PyG0. 

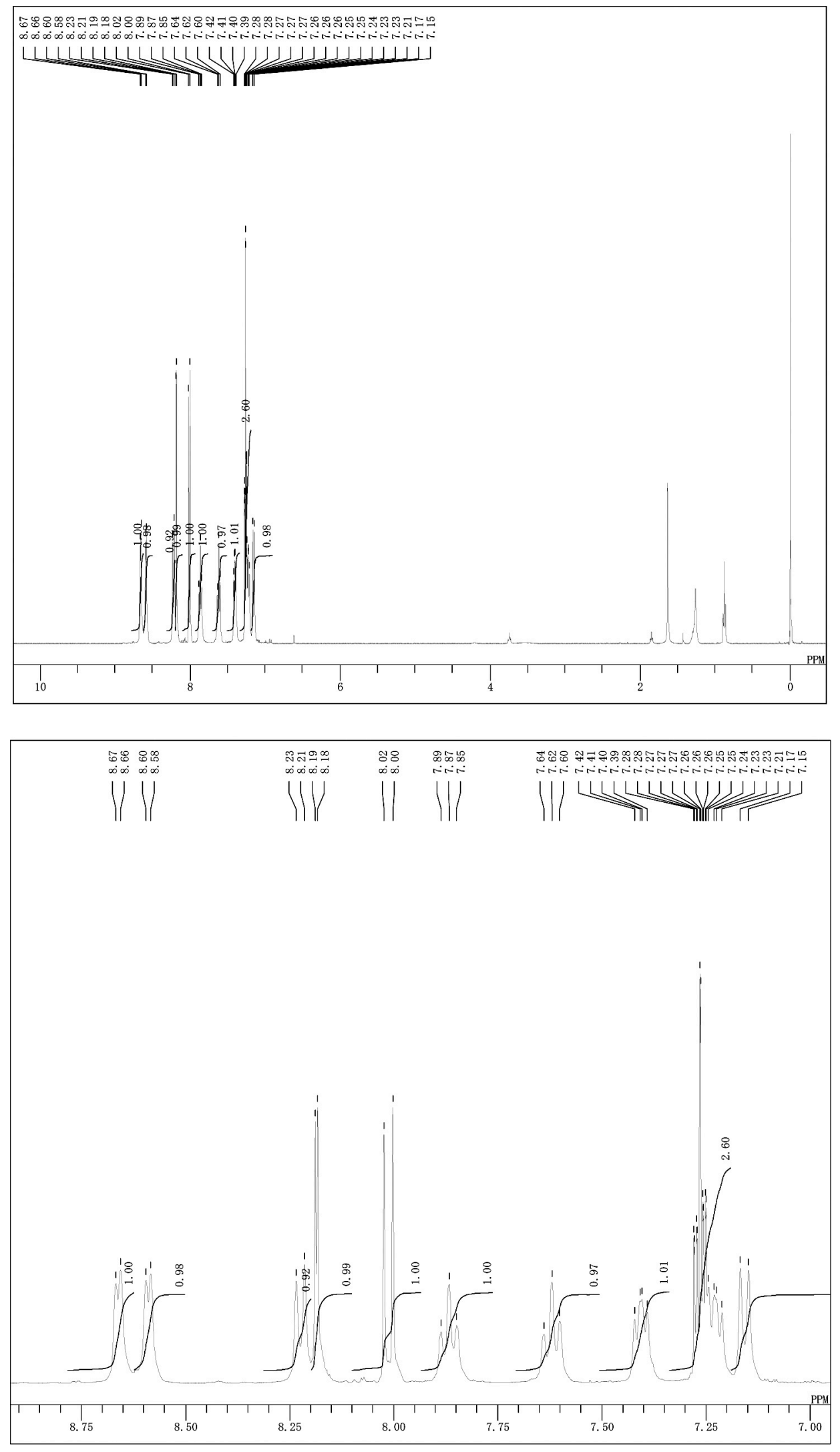

Figure S3. 1H NMR spectra of PyG2on. 


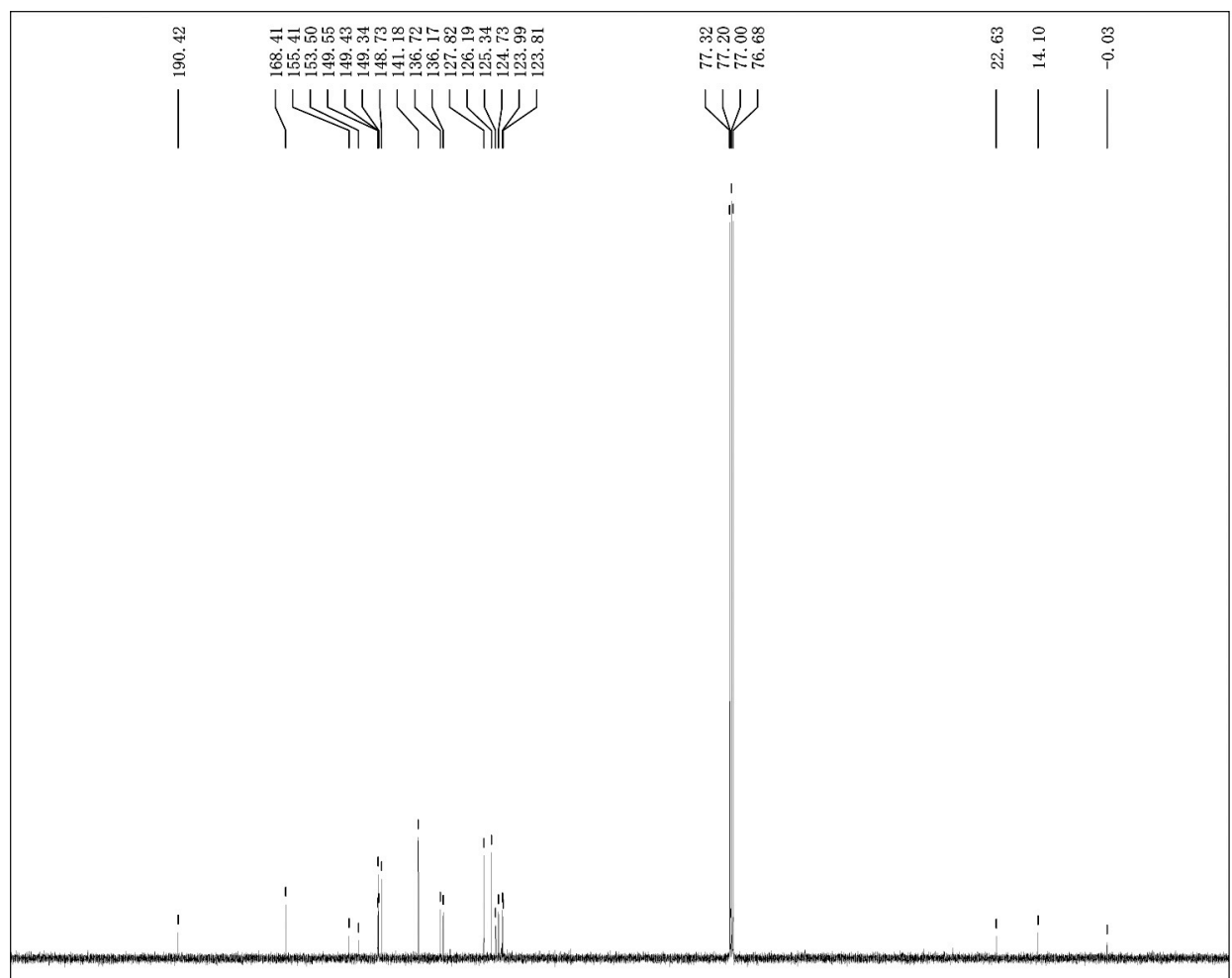

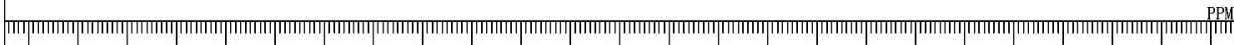
$\begin{array}{lllllllllllllllllllllllll}220 & 210 & 200 & 190 & 180 & 170 & 160 & 150 & 140 & 130 & 120 & 110 & 100 & 90 & 80 & 70 & 60 & 50 & 40 & 30 & 20 & 10 & 0 & -10 & -20\end{array}$

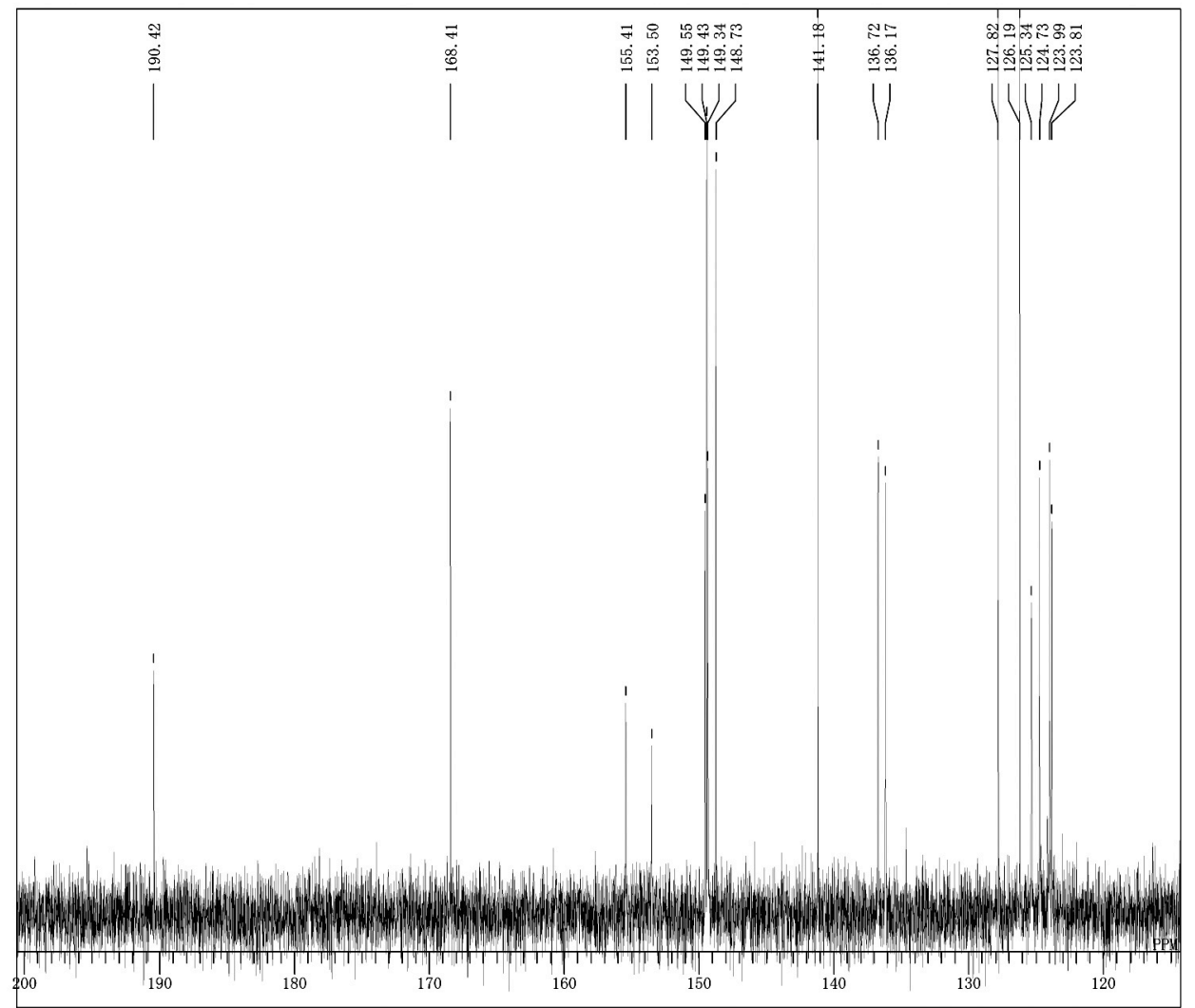

Figure S4. 13C NMR spectra of PyG2on. 

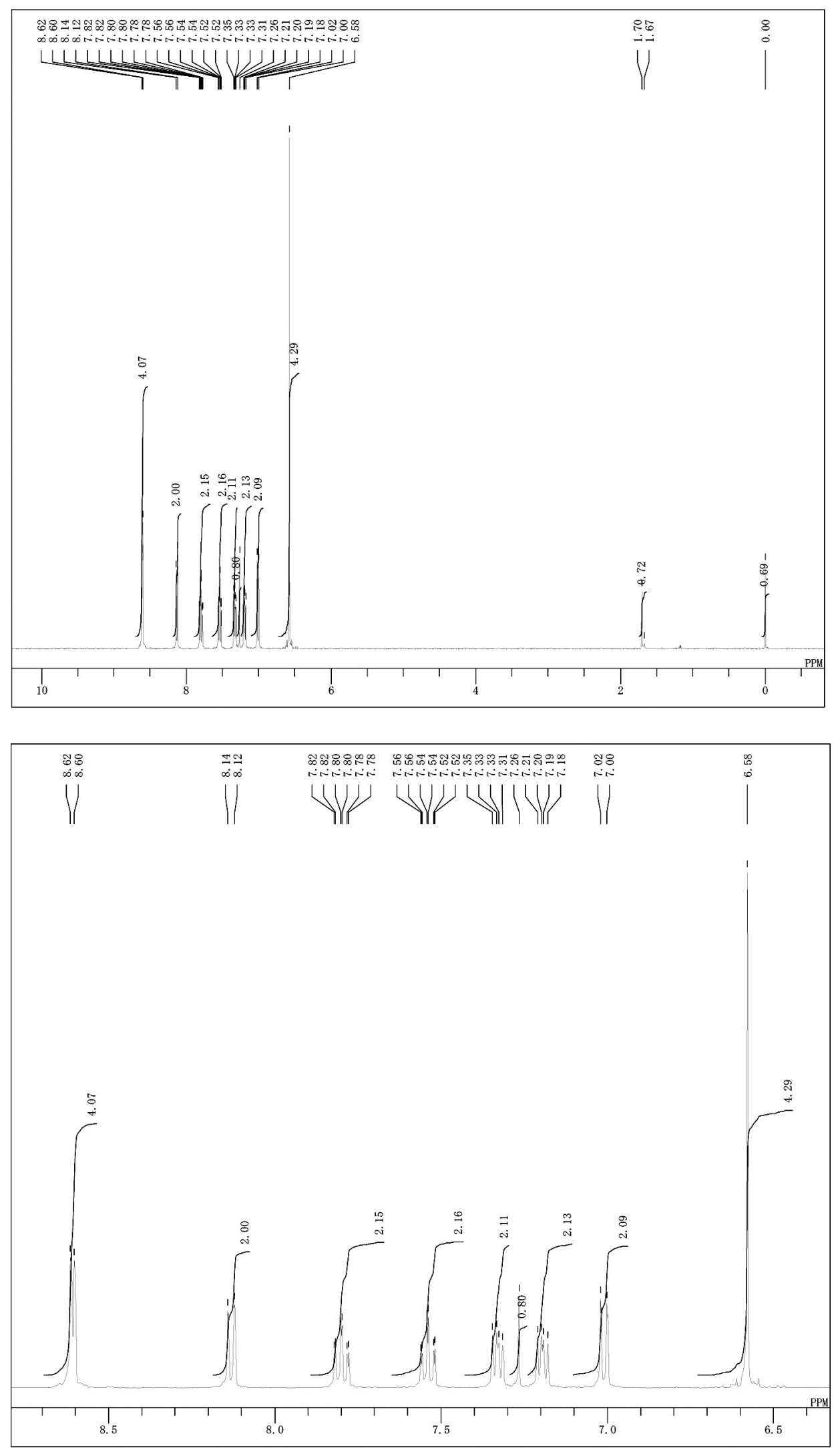

Figure S5. 1H NMR spectra of PyG1. 


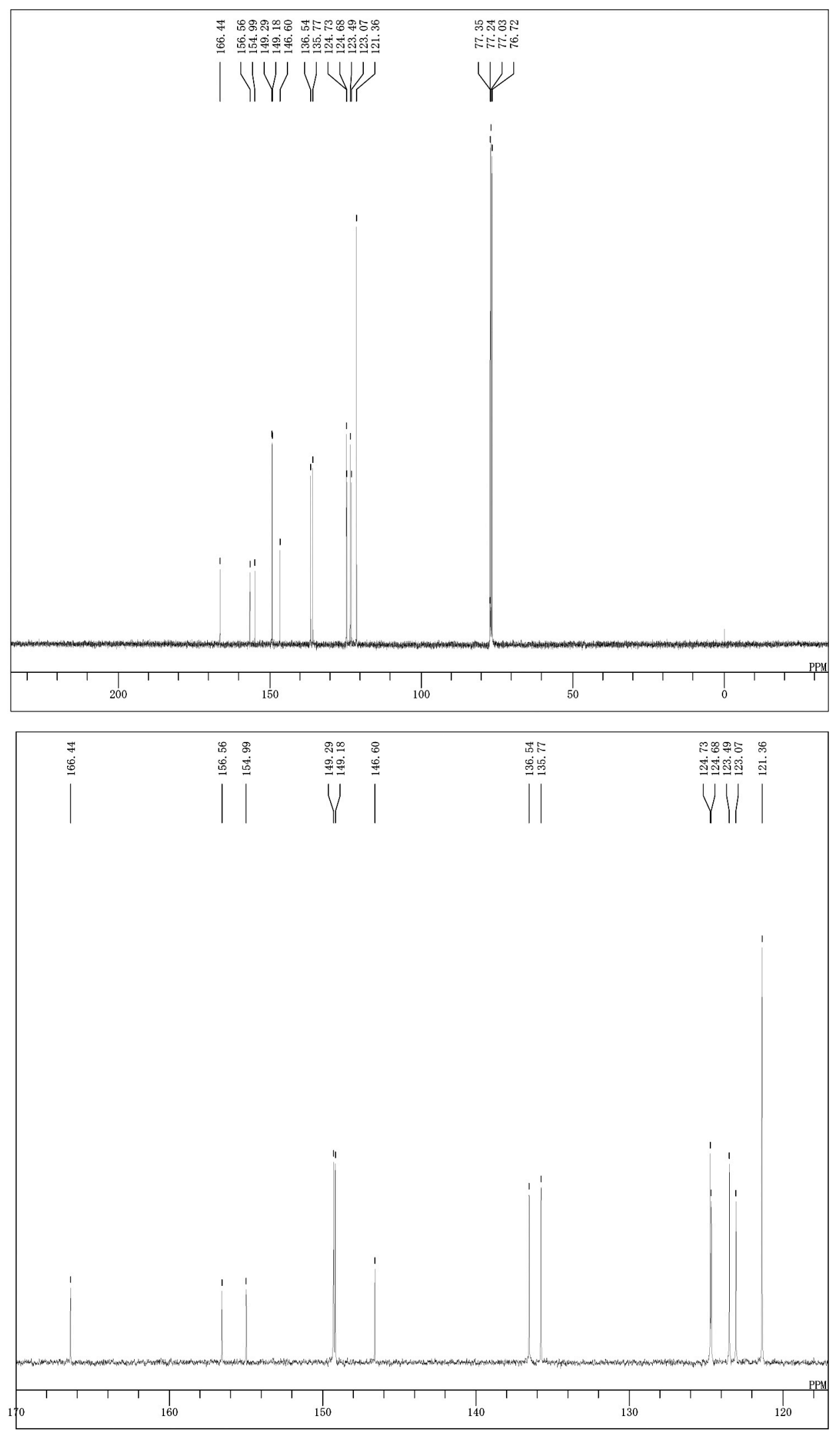

Figure S6. 13C NMR spectra of PyG1. 


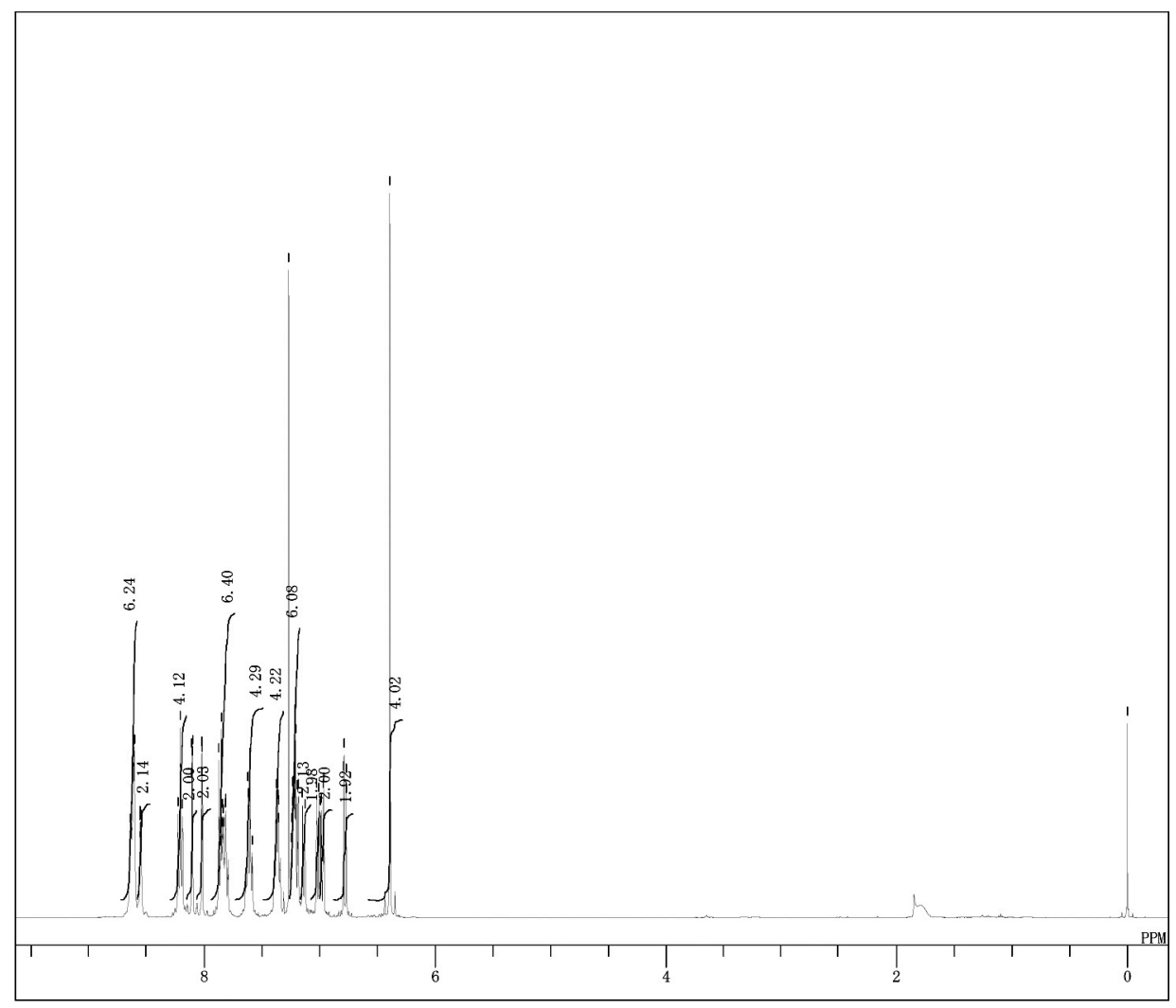

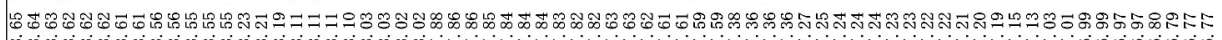

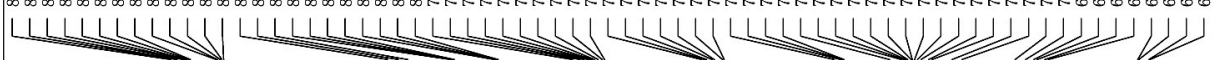

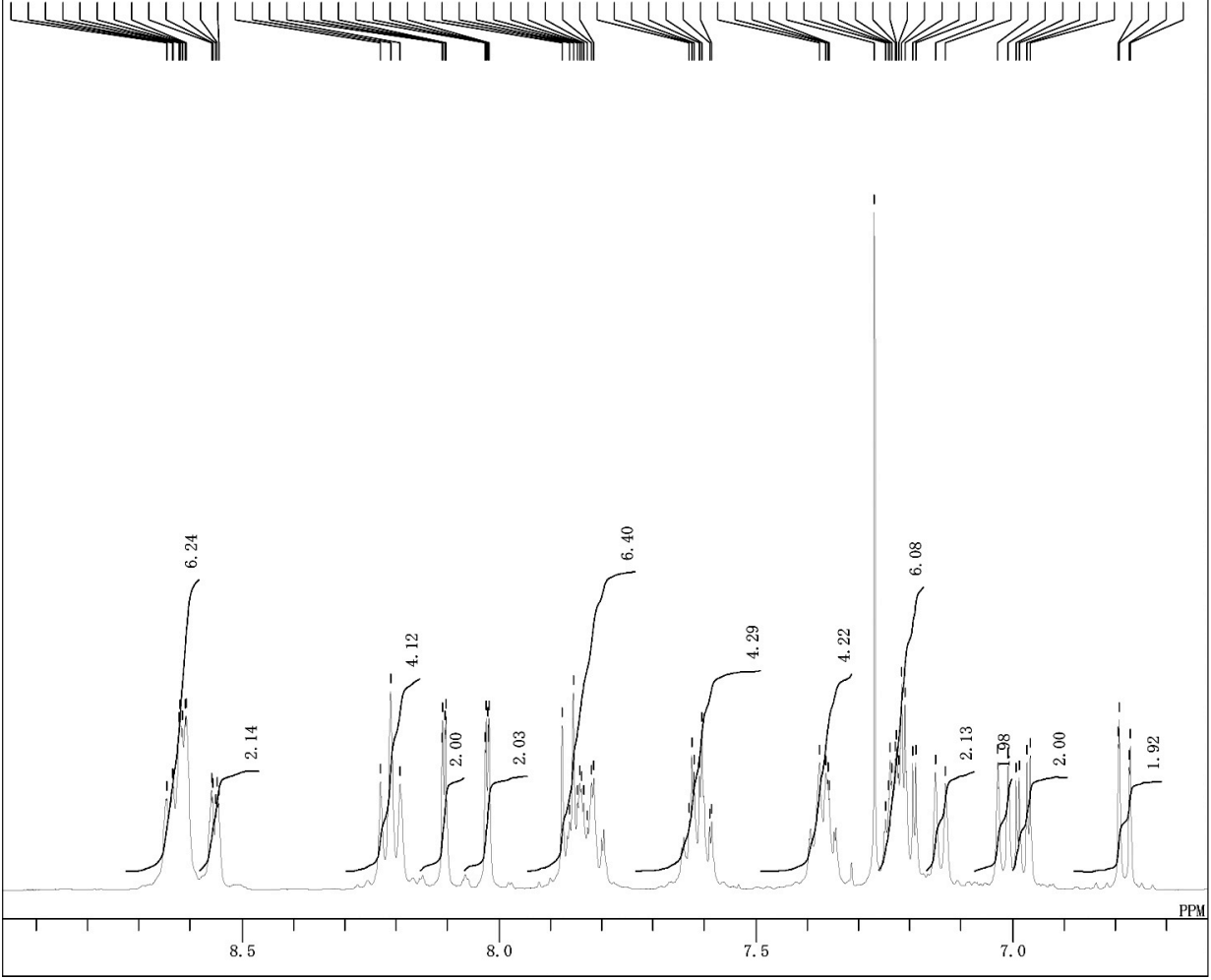

Figure S7. 1H NMR spectra of PyG2. 


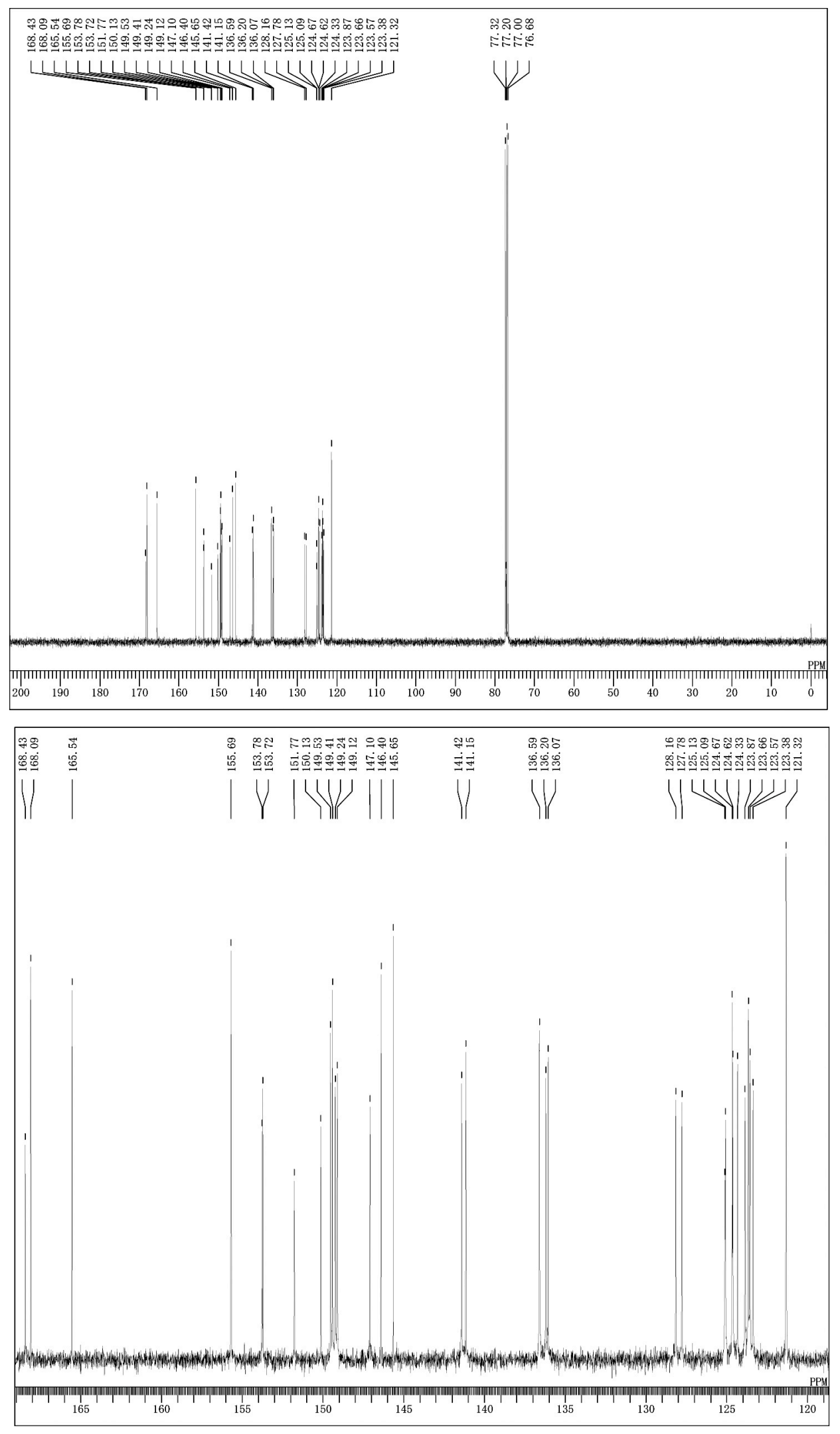

Figure S8. 13C NMR spectra of PyG2. 